\title{
On the absence of the one-sided Poincaré lemma in Cauchy-Riemann manifolds
}

\author{
FABIO NiCOLA
}

\begin{abstract}
Given an embeddable $C R$ manifold $M$ and a non-characteristic hypersurface $S \subset M$ we present a necessary condition for the tangential CauchyRiemann operator $\bar{\partial}_{M}$ on $M$ to be locally solvable near a point $x_{0} \in S$ in one of the sides determined by $S$.
\end{abstract}

Mathematics Subject Classification (2000): 32W10 (primary); 58J10 (secondary).

\section{Introduction and discussion of the results}

Let $M$ be a $C R$ manifold of type $(n, d)$ (so that $\operatorname{dim} M=2 n+d$ ). For an open subset $\mathcal{O} \subset M$ we denote by $C^{\infty}\left(\mathcal{O}, \Lambda^{p, q}\right)$ the space of smooth $(p, q)$ forms in $\mathcal{O}$, $0 \leq p \leq m, 0 \leq q \leq n, m=n+d$ (see e.g. Treves [16] and Section 2 below for terminology).

The Poincaré lemma is said to hold for the tangential Cauchy-Riemann complex $\bar{\partial}_{M}$ in degree $q, 1 \leq q \leq n$, at the point $x_{0} \in M$, if for every open neighborhood $\Omega$ of $x_{0}$ there is an open neighborhood $\Omega^{\prime} \subset \Omega$ such that the system

$$
\bar{\partial}_{M} u=f
$$

admits a solution $u \in C^{\infty}\left(\Omega^{\prime}, \Lambda^{0, q-1}\right)$ for all $f \in C^{\infty}\left(\Omega, \Lambda^{0, q}\right)$ which are cocycles, i.e. $\bar{\partial}_{M}$-closed (indeed we have $\bar{\partial}_{M}^{2}=0$, so that this condition is necessary). By a classical argument due to Grothendiek the definition given is in fact equivalent to the apparently weaker version of solvability in the sense of germs of smooth forms.

Necessary and sufficient conditions for the Poincaré lemma to hold have been object of investigation by many authors; we refer the reader, among others, to the important contributions by Lewy [10], Hörmander [8], Andreotti and Hill [2], Andreotti, Fredricks and Nacinovich [1], Folland and Stein [6], Nacinovich [13, 14], Michel [12], Treves [16, 18], Chen and Shaw [5], Hill and Nacinovich [7], Peloso 
and Ricci [15]. We also refer to Treves [17], Cordaro and Hounie [3] and the references therein for the study of the Poincare lemma in the more general context of involutive structures.

The present paper is devoted to the related question of finding necessary conditions for the local solvability of (1.1) in one side of a given smooth hypersurface $S \subset M$.

Precisely, we reason in an open neighborhood $\mathcal{O} \subset M$ of a given point $0 \in S$, say, the origin of the coordinates, and we suppose that $\mathcal{O} \cap S=\{\rho=0\}$, for a given smooth real function $\rho \in C^{\infty}(\mathcal{O} ; \mathbb{R})$, satisfying $d \rho \neq 0$ where $\rho=0$. Moreover, we adopt the following notation: for any open subset $\Omega \subset \mathcal{O}$ we define $\Omega_{-}=\Omega \cap\{\rho \leq 0\}$.

We allow distribution solutions and consider the following form of one-sided local solvability, which involves two open subsets $\Omega^{\prime} \subset \Omega \subset \mathcal{O}$ too:

$$
\begin{aligned}
& \text { Given any cocycle } f \in C^{\infty}\left(\Omega_{-}, \Lambda^{0, q}\right) \text { there is a distribution section } \\
& \qquad u \in \mathcal{A}^{\prime}\left(\Omega_{-}^{\prime}, \Lambda^{0, q-1}\right) \text { such that } \bar{\partial}_{M} u=f \text { in } \Omega_{-}^{\prime} .
\end{aligned}
$$

On a smooth manifold with boundary, we are denoting here by $\mathcal{A}^{\prime}$ the dual of the space of conormal distributions to the boundary (see Melrose [11], Treves [17] and Section 2 below). We have $C^{\infty} \subset \mathcal{A}^{\prime} \subset \mathcal{D}^{\prime}$ with dense inclusions. The choice of this space of distributions smaller than $\mathcal{D}^{\prime}$ is due to a difficulty in defining the action of a differential operator on a distribution because of the boundary term in the integration by parts formula. Instead, when $S$ inherits a $C R$ structure, for such a subspace there is a well defined restriction map $i^{*}: \mathcal{A}^{\prime}\left(\Omega_{-}^{\prime}, \Lambda^{p, q}\right) \rightarrow$ $\mathcal{D}^{\prime}\left(\partial \Omega_{-}^{\prime}, \Lambda_{b}^{p, q}\right)$ (the space of $(p, q)$-distribution sections on $\left.\partial \Omega_{-}^{\prime}=S \cap \Omega^{\prime}\right)$ which continuously extends the pull-back to the boundary of smooth forms. Hence, given $u \in \mathcal{A}^{\prime}\left(\Omega_{-}^{\prime}, \Lambda^{p, q}\right)$, the distribution section $\bar{\partial}_{M} u$ can be defined via the formula

$\left\langle\bar{\partial}_{M} u, \phi\right\rangle=(-1)^{p+q-1}\left\langle u, \bar{\partial}_{M} \phi\right\rangle+\left\langle i^{*} u, i^{*} \phi\right\rangle, \quad \forall \phi \in C_{c}^{\infty}\left(\Omega_{-}^{\prime}, \Lambda^{m-p, n-q-1}\right)$,

which agrees with the case in which $u \in C^{\infty}\left(\Omega_{-}^{\prime}, \Lambda^{p, q}\right)$.

We also observe that (1.2) is clearly different from the microlocal solvability (with respect to $\Omega_{-}$) in the sense of hyperfunctions, that is solvability in $\operatorname{int}\left(\Omega_{-}\right.$) within forms with arbitrary growth at $\partial \Omega_{-}$(see Kashiwara and Schapira [9], Michel [12], Cordaro and Treves [4]).

To establish our result we need a little terminology.

We denote by $T^{0,1} M \subset \mathbb{C} T M$ the $C R$ structure of $M$ and, following the notation in Treves $[16,17]$, by $T^{\prime} M \subset \mathbb{C} T^{*} M$ its orthogonal with respect to the duality between forms and vector fields. Moreover we set $T^{0} M=T^{\prime} M \cap T^{*} M$ for the so-called characteristic bundle of $M$ (of rank $d$ ).

We suppose that $M$ is locally embeddable (i.e. locally integrable) and that $S$ is non-characteristic with respect to the $C R$ structure of $M$ (Treves [17], Definition I.4.1), namely $\left.d \rho\right|_{p} \notin T_{p}^{0} M$ for every $p \in S \cap \mathcal{O}$. This implies that $S$ itself inherits a structure of (embeddable) $C R$ manifold of type $(n-1, d+1)$, where 
$T^{0,1} S=T^{0,1} M \cap \mathbb{C} T S$ (hence we can talk about the vector bundles $T^{\prime} S, T^{0} S$ and so on).

We finally recall that at any point $\left(x_{0}, \omega_{0}\right) \in T^{0} S, \omega_{0} \neq 0$, it is well defined a sesquilinear form $\mathcal{B}_{\left(x_{0}, \omega_{0}\right)}: T_{x_{0}}^{0,1} S \times T_{x_{0}}^{0,1} S \rightarrow \mathbb{C}$, by

$$
\mathcal{B}_{\left(x_{0}, \omega_{0}\right)}\left(\mathbf{v}_{1}, \mathbf{v}_{2}\right)=\left\langle\omega_{0},\left.\left[V_{1}, \overline{V_{2}}\right]\right|_{x_{0}} / 2 \iota\right\rangle \quad \mathbf{v}_{1}, \mathbf{v}_{2} \in T_{x_{0}}^{0,1} S,
$$

where $V_{1}$ and $V_{2}$ are smooth sections of $T^{0,1} S$ such that $\left.V_{1}\right|_{x_{0}}=\mathbf{v}_{1},\left.V_{2}\right|_{x_{0}}=\mathbf{v}_{2}$. The associated quadratic form $T_{x_{0}}^{0,1} S \ni \mathbf{v} \mapsto \mathcal{B}_{\left(x_{0}, \omega_{0}\right)}(\mathbf{v}, \mathbf{v})$, or $\mathcal{B}_{\left(x_{0}, \omega_{0}\right)}$ itself, is called Levi form of $S$ evaluated at $\left(x_{0}, \omega_{0}\right)$.

Theorem 1.1. Let $M$ be a locally embeddable $C R$ manifold of type $(n, d)$, and let $S \subset M$ be a non-characteristic smooth hypersurface. With the notation above, let us suppose that there exists $\omega_{0} \in T_{0}^{*} M$ such that $\omega_{0}+\left.\iota d \rho\right|_{0} \in T_{0}^{\prime} M$ and the Levi form of $S$ at $\left(0, \omega_{0}\right)$ is non degenerate and has exactly $q$ positive eigenvalues, $1 \leq q \leq n-1$.

Then there exists a neighborhood $\mathcal{O}$ of 0 such that (1.2) does not hold for any choice of the open neighborhoods $\Omega^{\prime} \subset \Omega \subset \mathcal{O}$ of 0 .

Indeed, if $\omega_{0} \in T_{0}^{*} M$ satisfies $\omega_{0}+\left.\iota d \rho\right|_{0} \in T_{0}^{\prime} M$, it is easily seen that the restriction $\left.\omega_{0}\right|_{T_{0} S}$ defines a non-zero element in $T_{0}^{0} S .{ }^{1}$ Hence the evaluation of the Levi form of $S$ at $\left(0, \omega_{0}\right)$ makes sense. Moreover the hypothesis is clearly independent of the choice of the defining function $\rho$ among those which preserve the orientation of $S$.

For the reader who is not interested in distribution solutions we observe that the condition in Theorem 1.1 is a fortiori necessary for the existence of smooth solutions, since $C^{\infty}\left(\Omega_{-}^{\prime}, \Lambda^{0, q-1}\right) \subset \mathcal{A}^{\prime}\left(\Omega_{-}^{\prime}, \Lambda^{0, q-1}\right)$. However, the absence of smooth solutions, under hypotheses equivalent to the ones in Theorem 1.1, was already established by Nacinovich in [14] (see the third assertion in Proposition 9, page 481).

When $M$ is a complex manifold, i.e. $d=0$, results about the one-sided local solvability of $\bar{\partial}_{M}$ were known from Andreotti and Hill [2] and Michel [12]. In this special case our result agrees with Theorem 4 of [2], page 798. Indeed it is proved there that, if the Hermitian form $\sum_{j, k=1}^{n} \partial^{2} \rho /\left.\partial z_{j} \partial \bar{z}_{k}\right|_{0} w_{j} \bar{w}_{k}$ restricted to $T_{0}^{0,1} S$ is non degenerate and has exactly $q$ negative eigenvalues then there is a neighborhood $\mathcal{O}$ of 0 such that, for every domain of holomorphy $\Omega \subset \mathcal{O}$ the cohomology spaces $H_{\bar{\partial}_{M}}^{0, q}\left(\Omega_{-}\right.$) are infinite dimensional (pay attention to the different convention of sign in the definition of the Levi form; here we decided to follow [16, 17]). As we observed, this result was then generalized in [14] when $M$ is a $C R$ manifold.

${ }^{1}$ We have $\left.d \rho\right|_{0}(\mathbf{v})=0$ if $\mathbf{v} \in \mathbb{C} T S$. Hence $\omega_{0}(\mathbf{v})=0$ if $\mathbf{v} \in T^{0,1} S$ because any covector in $T_{0}^{\prime} M$ vanishes on such a subspace too. Therefore $\left.\omega_{0}\right|_{T_{0}} S \in T_{0}^{0} S$. Finally $\omega_{0} \mid T_{0} S \not \equiv 0$, for otherwise there would exist $\alpha \in \mathbb{R}$ such that $\omega_{0}=\left.\alpha d \rho\right|_{0}$, and therefore $\left.(\alpha+\imath) d \rho\right|_{0} \in T_{0}^{\prime} M$, which contradicts the fact that $S$ is non-characteristic. 
We finally notice that, under the hypotheses in Theorem 1.1 the Poincare lemma for the $\bar{\partial}_{S}$ necessarily fails in degree $q$ at 0 in view of the classical results by Andreotti, Fredricks and Nacinovich [1]. On the other hand, the Poincaré lemma for the $\bar{\partial}_{M}$ can hold true or not, for, e.g. the signature of the Levi form of $M$ at characteristic points $\left(0, \omega_{0}\right) \in T^{0} M$ can be arbitrary. This shows that Theorem 1.1 cannot be deduced from the necessary condition in [1] for the existence of Poincaré lemma for the $\bar{\partial}_{S}$ combined with a Mayer-Vietoris argument (cf. [2], Part I, and [17]). We refer to Section 4 for an example related to this fact and also for a few words about the top degree case $(q=n)$, which is not treated in Theorem 1.1.

Acknowledgments. I wish to thank Marco M. Peloso and Luigi Rodino for helpful discussions and Mauro Nacinovich for pointing out his paper [14].

\section{Preliminaries}

In this section we fix the notation used in this paper; we follow $[16,17]$.

By definition, a $C R$ structure of type $(n, d)$ on a smooth real manifold $M$, $\operatorname{dim} M=2 n+d$ is defined by a subbundle $T^{0,1} M \subset \mathbb{C} T M$ of rank $n$ such that $T^{0,1} M \cap \overline{T^{0,1} M}=0$ and satisfying the Frobenius formal integrability condition. We say that $M$ is locally embeddable (or locally integrable) if every point of $M$ has an open neighborhood $\mathcal{O}$ such that the bundle $T^{\prime} M:=\left\{(x, \alpha) \in \mathbb{C} T^{*} M\right.$ : $\left.\alpha(\mathbf{v})=0, \forall \mathbf{v} \in T_{x}^{0,1} M\right\}$ (of rank $m=n+d$ ) is spanned on $\mathcal{O}$ by exact forms $d Z_{1}, \ldots, d Z_{m}$. The functions $Z_{1}, \ldots, Z_{m}$ are then called a system of first integrals. We denote by $T^{\prime p, q} M$ the homogeneous part of degree $p+q$ in the ideal generated by the $p$-th exterior power of $T^{\prime} M$, i.e. an element $\zeta \in T_{x_{0}}^{\prime p, q} M$ has the form $\zeta_{1} \wedge \ldots \wedge \zeta_{p+q}$ where at least $p$ of the factors $\zeta \in \mathbb{C} T_{x_{0}}^{*} M$ belong to $T_{x_{0}}^{\prime} M$. Clearly $T^{\prime p+1, q-1} M \subset T^{p, q} M$, so that one can set $\Lambda^{p, q}=T^{p, q} M / T_{x_{0}}^{p+1, q-1} M$. As a consequence of the Frobenius condition it turns out that the differential defines a map $d: C^{\infty}\left(M, T^{\prime p, q} M\right) \rightarrow C^{\infty}\left(M, T^{\prime p, q+1} M\right)$ and consequently it induces an operator

$$
\bar{\partial}_{M}: C^{\infty}\left(M, \Lambda^{p, q}\right) \rightarrow C^{\infty}\left(M, \Lambda^{p, q+1}\right),
$$

which is called the tangential Cauchy-Riemann operator on $M$. In the sequel the elements of $C^{\infty}\left(M, \Lambda^{p, q}\right)$ will be referred to as forms instead of equivalence classes of forms, when there is not risk of misunderstanding.

We already recalled in the introduction the definition of the Levi form at a characteristic point $\omega_{0} \in T_{x_{0}}^{0} M:=T_{x_{0}}^{\prime} M \cap T_{x_{0}}^{*} M$ (we emphasize that in Theorem 1.1 we use the Levi form of $S$, which inherits a structure of $C R$ manifold of type $(n-1, d+1)$ by defining $\left.T^{0,1} S:=T^{0,1} M \cap \mathbb{C} T S\right)$.

We now want to say a few words about the spaces of distributions involved in (1.2) (see [11] and especially [17]). 
We use the notation introduced there, so $\Omega^{\prime} \subset M$ is an open neighborhood of $0 \in S \subset M$, and $\Omega_{-}^{\prime}=\Omega^{\prime} \cap\{\rho \leq 0\}$, regarded as a manifold with boundary. Then we denote by $\mathcal{D}^{\prime}\left(\Omega_{-}^{\prime}, \Lambda^{p, q}\right)$ the dual of the space $C_{c}^{\infty}\left(\Omega_{-}^{\prime}, \Lambda^{m-p, n-q}\right)$ of smooth forms, compactly supported in $\Omega_{-}^{\prime}$ (the support can of course intersect the boundary $\Omega^{\prime} \cap S$ of $\left.\Omega_{-}^{\prime}\right)$. We regard $C^{\infty}\left(\Omega_{-}^{\prime}, \Lambda^{p, q}\right)$ as a subspace of $\mathcal{D}^{\prime}\left(\Omega_{-}^{\prime}, \Lambda^{p, q}\right)$ via the pairing

$$
\langle u, \phi\rangle=\int_{\Omega_{-}^{\prime}} u \wedge \phi, \quad u \in C^{\infty}\left(\Omega_{-}^{\prime}, \Lambda^{p, q}\right), \phi \in C_{c}^{\infty}\left(\Omega_{-}^{\prime}, \Lambda^{m-p, n-q}\right),
$$

which is well defined (i.e. independently of the representatives).

For $s \in \mathbb{R}$ we define $\dot{H}_{l o c}^{s}\left(\Omega_{-}^{\prime}, \Lambda^{p, q}\right)$ as the closed subspace of $H_{l o c}^{s}\left(\Omega^{\prime}, \Lambda^{p, q}\right)$ of all distribution sections whose support is contained in $\Omega_{-}^{\prime}$. Moreover one denotes by $\dot{\mathcal{A}}^{(s)}\left(\Omega_{-}^{\prime}, \Lambda^{p, q}\right)$ the space of all $u \in \dot{H}_{l o c}^{s}\left(\Omega_{-}^{\prime}, \Lambda^{p, q}\right)$ such that $P u \in$ $\dot{H}_{l o c}^{s}\left(\Omega_{-}^{\prime}, \Lambda^{p, q}\right)$ for all totally characteristic operators $P$, i.e. differential operators in the algebra generated by the vector fields tangent to the boundary of $\Omega_{-}^{\prime}$ ( $P$ acts on distributions, as usual, via its transpose, which still is a totally characteristic operator). We equip $\dot{\mathcal{A}}^{(s)}\left(\Omega_{-}^{\prime}, \Lambda^{p, q}\right)$ with the coarest locally convex topology that ensures the continuity of all maps $\dot{\mathcal{A}}^{(s)}\left(\Omega_{-}^{\prime}, \Lambda^{p, q}\right) \rightarrow P u \in \dot{H}_{l o c}^{s}\left(\Omega_{-}^{\prime}, \Lambda^{p, q}\right)$, so that $\dot{H}_{l o c}^{s}\left(\Omega_{-}^{\prime}, \Lambda^{p, q}\right)$ is a Fréchet space. Then one also considers the closed subspaces $\dot{\mathcal{A}}^{(s)}\left(K, \Lambda^{p, q}\right)$, with $K \subset \Omega_{-}^{\prime}$ compact. Finally we define $\dot{\mathcal{A}}_{c}^{(s)}\left(\Omega_{-}^{\prime}, \Lambda^{p, q}\right)$ as the inductive limit of $\dot{\mathcal{A}}^{(s)}\left(K, \Lambda^{p, q}\right)$, with $K \subset \Omega_{-}^{\prime}$ compact.

If $s \leq 0$, we have $C^{\infty}\left(\Omega_{-}^{\prime}, \Lambda^{p, q}\right) \subset \dot{\mathcal{A}}^{(s)}\left(\Omega_{-}^{\prime}, \Lambda^{p, q}\right)$ and $C_{c}^{\infty}\left(\Omega_{-}^{\prime}, \Lambda^{p, q}\right)$ is dense in $\dot{\mathcal{A}}_{c}^{(s)}\left(\Omega_{-}^{\prime}, \Lambda^{p, q}\right)$ and $\dot{\mathcal{A}}^{(s)}\left(\Omega_{-}^{\prime}, \Lambda^{p, q}\right)$.

Definition 2.1. We denote by $\mathcal{A}^{\prime}\left(\Omega_{-}^{\prime}, \Lambda^{p, q}\right)$ the subspace of distribution sections $u \in \mathcal{D}^{\prime}\left(\Omega_{-}^{\prime}, \Lambda^{p, q}\right)$ such that, for every $s \leq 0$, the functional $\phi \mapsto\langle u, \phi\rangle$ extends continuously from $C_{c}^{\infty}\left(\Omega_{-}^{\prime}, \Lambda^{p, q}\right)$ to $\dot{\mathcal{A}}^{(s)}\left(\Omega_{-}^{\prime}, \Lambda^{p, q}\right)$.

We have $C^{\infty}\left(\Omega_{-}^{\prime}, \Lambda^{p, q}\right) \subset \mathcal{A}^{\prime}\left(\Omega_{-}^{\prime}, \Lambda^{p, q}\right) \subset \mathcal{D}^{\prime}\left(\Omega_{-}^{\prime}, \Lambda^{p, q}\right)$.

As anticipated in the introduction, there is a well defined continuous linear map $i^{*}: \mathcal{A}^{\prime}\left(\Omega_{-}^{\prime}, \Lambda^{p, q}\right) \rightarrow \mathcal{D}^{\prime}\left(\partial \Omega_{-}^{\prime}, \Lambda_{b}^{p, q}\right)$ which extends the pull-back of smooth forms to the boundary. Precisely such a map is defined by

$$
\left\langle i^{*} u, \phi\right\rangle=(-1)^{p+q}\langle u, E \phi\rangle, \quad u \in \mathcal{A}^{\prime}\left(\Omega_{-}^{\prime}, \Lambda^{p, q}\right), \phi \in C_{c}^{\infty}\left(\partial \Omega_{-}^{\prime}, \Lambda_{b}^{m-p, n-q-1}\right),
$$

where $E: \mathcal{D}^{\prime}\left(\partial \Omega_{-}^{\prime}, \Lambda_{b}^{p, q}\right) \rightarrow \mathcal{D}^{\prime}\left(\Omega_{-}^{\prime}, \Lambda^{p, q+1}\right),\langle E \psi, \phi\rangle=\left\langle\psi, i^{*} \phi\right\rangle,\left(i^{*} \phi\right.$ is the usual pull-back to the boundary of $\left.\phi \in C_{c}^{\infty}\left(\Omega_{-}^{\prime}, \Lambda^{m-p, n-q-1}\right)\right)$. This makes sense since one can prove that $E \psi \in \dot{\mathcal{A}}^{(-1)}\left(\Omega_{-}^{\prime}, \Lambda^{p, q}\right)$ if $\psi \in C_{c}^{\infty}\left(\partial \Omega_{-}^{\prime}, \Lambda^{m-p, n-q-1}\right)$. As a consequence, for $u \in \mathcal{A}^{\prime}\left(\Omega_{-}^{\prime}, \Lambda^{p, q}\right)$ one can define the distribution section $\bar{\partial}_{M} u$ as in (1.3), and this extends the usual action when $u \in C^{\infty}\left(\Omega_{-}^{\prime}, \Lambda^{p, n-q}\right)$. 


\section{An a priori estimate}

In this section we assume (1.2) and we prove an a priori inequality by means of arguments from Functional Analysis. In the next section we will show that such an estimate cannot hold under the assumptions of Theorem 1.1. Indeed this is the classical pattern to prove necessary conditions for local solvability; see e.g. [8, 1, 17].

For an open subset $V \subset M$ we set

$$
\mathcal{J}_{c}^{\infty}\left(V_{-}, \Lambda^{p, q}\right)=\left\{v \in C_{c}^{\infty}\left(V_{-}, \Lambda^{p, q}\right), i^{*}(v)=0\right\} .
$$

Moreover we denote by $\|\cdot\|_{K, l}$, with $K \subset M$ compact and $l \in \mathbb{Z}_{+}$, the seminorms which define the topology of $C^{\infty}\left(M, \Lambda^{p, q}\right)$.

Proposition 3.1. Assume (1.2). Then for every compact subset $K^{\prime} \subset \Omega_{-}^{\prime}$ there exist a compact $K \subset \Omega_{-}$and constants $C>0, l \in \mathbb{Z}_{+}$such that, for every cocycle $f \in C^{\infty}\left(\Omega_{-}, \Lambda^{0, q}\right)$ and every $v \in \mathcal{J}_{c}^{\infty}\left(\Omega_{-}^{\prime}, \Lambda^{m, n-q}\right)$ with supp $v \subset K^{\prime}$ we have

$$
\left|\int_{\Omega_{-}} f \wedge v\right| \leq C\|f\|_{K, l}\left\|\bar{\partial}_{M} v\right\|_{K^{\prime}, l} .
$$

Proof. We set $E=\operatorname{Ker}\left\{\bar{\partial}_{M}: C^{\infty}\left(\Omega_{-}, \Lambda^{0, q}\right) \rightarrow C^{\infty}\left(\Omega_{-}, \Lambda^{0, q+1}\right)\right\}$, with the topology inherited from $C^{\infty}\left(\Omega_{-}, \Lambda^{0, q}\right)$, and $F=\left\{v \in \mathcal{J}_{c}^{\infty}\left(\Omega_{-}^{\prime}, \Lambda^{m, n-q}\right)\right.$, supp $v \subset$ $K^{\prime}$ \}, with the topology given by the seminorms $\left\|\bar{\partial}_{M} v\right\|_{K^{\prime}, l}$. $E$ is a Fréchet space, whereas $F$ is not Hausdorff if $q<n$. Hence we also consider the associated Hausdorff space $F_{0}=F / \overline{\{0\}}$, where the closure of $\{0\}$ consists of the cocycles that belong to $\mathcal{J}_{c}^{\infty}\left(\Omega_{-}^{\prime}, \Lambda^{m, n-q}\right)$.

We observe that the bilinear functional

$$
E \times F \ni(f, v) \longmapsto \int_{\Omega_{-}} f \wedge v
$$

is certainly continuous for every fixed $v \in F$. On the other hand, for any fixed $f \in E$, by assumption there exists $u \in \mathcal{A}^{\prime}\left(\Omega_{-}^{\prime}, \Lambda^{0, q-1}\right)$ satisfying $\bar{\partial}_{M} u=f$ in $\Omega_{-}^{\prime}$, namely

$$
\left\langle\bar{\partial}_{M} u, \phi\right\rangle:=(-1)^{p+q}\left\langle u, \bar{\partial}_{M} \phi\right\rangle+\left\langle i^{*} u, i^{*} \phi\right\rangle=\int_{\Omega_{-}} f \wedge \phi, \quad \forall \phi \in C_{c}^{\infty}\left(\Omega_{-}^{\prime}, \Lambda^{m, n-q}\right) .
$$

In particular, if $\phi=v \in \mathcal{J}_{c}^{\infty}\left(\Omega_{-}^{\prime}, \Lambda^{m, n-q}\right)$ we have $i^{*} v=0$, and we deduce that the functional (3.2) is separately continuous and induces a separately continuous bilinear functional on $E \times F_{0}$. Since $E$ is Fréchet and $F_{0}$ is metrizable it follows that it is in fact continuous, and so is its lift to $E \times F$. But this is exactly the meaning of (3.1). 


\section{Proof of Theorem 1.1}

The proof of Theorem 1.1 is based on the use of a special system of coordinates whose existence is shown in the following proposition.

Proposition 4.1. Under the assumptions of Theorem 1.1, in a neighborhood of 0 in $M$ there exist coordinates $x_{j}, y_{j}$ and $s_{k}, j=1, \ldots, n, k=1, \ldots, d$, such that a system of first integrals for the $C R$ structure on $M$ is given by

$$
\begin{array}{rlrl}
z_{j} & =x_{j}+\iota y_{j}, & j & =1, \ldots, n, \\
w_{k}:=s_{k}+\iota \phi_{k}(z, s), & k & =1, \ldots, d,
\end{array}
$$

for convenient smooth real functions $\phi_{k}$ satisfying

$$
\phi_{k}(0)=0,\left.\quad d \phi_{k}\right|_{0}=0 .
$$

Moreover there exists another defining function $\rho^{\prime}(z, s)$ for $S$ near 0 satisfying $\left.d \rho^{\prime}\right|_{0}=\left.d \rho\right|_{0}$ and, upon setting $z^{\prime}=\left(z_{1}, \ldots, z_{n-1}\right)$,

$$
\rho^{\prime}(z, s)=y_{n}-\phi_{0}\left(z^{\prime}, x_{n}, s\right),
$$

with

$$
\phi_{0}\left(z^{\prime}, x_{n}, s\right)=\sum_{j=1}^{q}\left|z_{j}\right|^{2}-\sum_{j=q+1}^{n-1}\left|z_{j}\right|^{2}+O\left(\left|z^{\prime}\right|^{3}+\left|x_{n}\right|^{3}+|s|^{3}\right) .
$$

Proof. We know e.g. from Treves $[16,17]$ that on any locally embeddable $C R$ manifold of type $(n, d)$ there exist coordinates $x_{j}, y_{j}, s_{k}$ for which $z_{j}$ and $w_{k}$ in (4.1) and (4.2) are a system of first integrals, for convenient $\phi_{k}$ satisfying (4.3). In such a system of coordinates we have $T_{0}^{0,1} M=\operatorname{span}_{\mathbb{C}}\left\{\left.\frac{\partial}{\partial \bar{z}_{j}}\right|_{0} ; j=1, \ldots, n\right\}, T_{0}^{\prime} M=$ $\operatorname{span}_{\mathbb{C}}\left\{\left.d z_{j}\right|_{0},\left.d s_{k}\right|_{0} ; j=1, \ldots, n, k=1, \ldots, d\right\}$, and $T_{0}^{0} M=\operatorname{span}_{\mathbb{R}}\left\{\left.d s_{k}\right|_{0} ; k=\right.$ $1, \ldots, d\}$.

By assumption we have $\left.d \rho\right|_{0} \notin T_{0}^{\prime} M$. Hence we have, say, $\left.\frac{\partial \rho}{\partial \bar{z}_{n}}\right|_{0} \neq 0$. We can therefore replace $z_{n}$ by a suitable linear combination $\tilde{z}_{n}$ of $z_{j}$ and $w_{k}, j=$ $1, \ldots, n, k=1, \ldots, d$ in such a way that, by taking $\tilde{x}_{n}=\operatorname{Re} z_{n}$ and $\tilde{y}_{n}=\operatorname{Im} z_{n}$ as new coordinates together with $x^{\prime}:=\left(x_{1}, \ldots, x_{n-1}\right), y^{\prime}:=\left(y_{1}, \ldots, y_{n-1}\right)$, $s=\left(s_{1}, \ldots, s_{d}\right)$, and after deleting the tildes we have

$$
\rho=y_{n}-\psi(z, s),
$$

for a suitable smooth real function $\psi$ satisfying $\psi(0)=0,\left.d \psi\right|_{0}=0 .^{2}$ An application of the implicit function theorem shows that $S$ can be defined near 0 by a

${ }^{2}$ Indeed, we have $\left.d \rho\right|_{0}=\sum_{j=1}^{n}\left(\left.a_{j} d x_{j}\right|_{0}+\left.a_{n}^{\prime} d y_{j}\right|_{0}\right)+\left.\sum_{k=1}^{d} c_{k} d s_{k}\right|_{0}$, with $a_{j}, a_{j}^{\prime}$ and $c_{k}$ real numbers and $a_{n}^{2}+a_{n}^{\prime 2} \neq 0$. Then it suffices to set $\tilde{z}_{n}=\sum_{j=1}^{n}\left(a_{j}^{\prime}+\iota a_{j}\right) z_{j}+\sum_{k=1}^{d} \iota c_{k} w_{k}$. The linear transformation $\left(x^{\prime}, y^{\prime}, x_{n}, y_{n}, s\right) \mapsto\left(x^{\prime}, y^{\prime}, \tilde{x}_{n}, \tilde{y}_{n}, s\right)$ is invertible, since its determinant equals $a_{n}^{2}+a_{n}^{\prime 2}$. 
function $\rho^{\prime}$ of the form

$$
\rho^{\prime}=y_{n}-\phi_{0}\left(z^{\prime}, x_{n}, s\right),
$$

for a convenient smooth real function $\phi_{0}$ satisfying $\phi_{0}(0)=0$ and $\left.d \phi_{0}\right|_{0}=0$. Hence we see that $\left.d \rho^{\prime}\right|_{0}=\left.d \rho\right|_{0}=\left.d y_{n}\right|_{0}$.

Now, as coordinates on $S$ we take the restrictions of $x_{j}, y_{j}$ and $s_{k}, j=$ $1, \ldots, n-1, k=1, \ldots, d$. A system of first integrals for the $C R$ structure induced on $S$ (of type $(n-1, d+1)$ ) is given by $z_{j}=x_{j}+\iota y_{j}, j=1, \ldots, n-1$, $z_{n}=x_{n}+\iota \phi_{0}\left(z^{\prime}, x_{n}, s\right)$ and $w_{k}=s_{k}+\left.\iota \phi_{k}\left(z^{\prime}, x_{n}, y_{n}, s\right)\right|_{y_{n}=\phi_{0}\left(z^{\prime}, x_{n}, s\right)}, k=1, \ldots, d$. By assumption there exists $\omega_{0} \in \operatorname{span}_{\mathbb{R}}\left\{\left.d x_{j}\right|_{0},\left.d y_{j}\right|_{0},\left.d s_{k}\right|_{0} ; j=1, \ldots, n, k=\right.$ $1, \ldots, d\}$ such that $\omega_{0}+\left.\iota d y_{n}\right|_{0} \in T_{0}^{\prime} M$. This implies that $\omega_{0}=\left.d x_{n}\right|_{0}+\left.\sum_{k=1}^{d} c_{k} d s_{k}\right|_{0}$, for certain $c_{k} \in \mathbb{R}$. In particular we see that $\omega_{0} \in T_{0}^{0} S=\operatorname{span}_{\mathbb{R}}\left\{\left.d x_{n}\right|_{0},\left.d s_{k}\right|_{0} ; k=\right.$ $1, \ldots, d\}$. It follows from [16] that the Levi form of $S$ at $\left(0, \omega_{0}\right)$, expressed in the basis $\left\{\left.\frac{\partial}{\partial \bar{z}_{j}}\right|_{0} ; j=1, \ldots, n-1\right\}$ of $T_{0}^{0,1} S$ is the matrix

$$
\left.\frac{\partial^{2}}{\partial \bar{z}_{i} \partial z_{j}}\left(\phi_{0}+\sum_{k=1}^{d} c_{k} \phi_{k}\right)\right|_{0}
$$

So, by setting $\tilde{z}_{n}:=z_{n}+\sum_{k=1}^{d} c_{k} w_{k}$ and taking $\tilde{x}_{n}=\operatorname{Re} \tilde{z}_{n}$ and $\tilde{y}_{n}=\operatorname{Im} \tilde{z}_{n}$ as new coordinates in place of $x_{n}, y_{n}$ we have $\left(\omega_{0}=\left.d \tilde{x}_{n}\right|_{0}\right.$ and $)$

$$
\rho^{\prime}=\tilde{y}_{n}-\tilde{\phi}_{0}\left(z^{\prime}, \tilde{x}_{n}, s\right)
$$

where $\tilde{\phi}_{0}:=\phi_{0}+\sum_{k=1}^{d} c_{k} \phi_{k}$, expressed in these coordinates. As a consequence of the assumption on the signature of the Levi form (4.5), we see that there exists a linear change of coordinates $\tilde{z}_{i}=b_{i j} z_{j}, i, j=1, \ldots, n-1$ such that, upon setting $\tilde{x}_{j}=\operatorname{Re} \tilde{z}_{j}, \tilde{y}_{j}=\operatorname{Im} \tilde{z}_{j}, j=1, \ldots, n-1$,

$$
\tilde{\phi}_{0}\left(\tilde{z}^{\prime}, \tilde{x}_{n}=0, s=0\right)=\sum_{j=1}^{q}\left|\tilde{z}_{j}\right|^{2}-\sum_{j=q+1}^{n-1}\left|\tilde{z}_{j}\right|^{2}+O\left(\left|\tilde{z}^{\prime}\right|^{3}\right) .
$$

Summing up, (after deleting the tildes) we found coordinates $x_{j}, y_{j}, s_{k}, j=1, \ldots, n$, $k=1, \ldots, d$, such that a system of first integrals is given by $z_{j}, w_{k}$ in (4.1) and (4.2), where $\phi_{k}$ satisfy (4.3). Moreover we found a defining function $\rho^{\prime}=y_{n}-$ $\phi_{0}\left(z^{\prime}, x_{n}, s\right)$ for $S$ near 0 , with

$$
\begin{aligned}
\phi_{0}\left(z^{\prime}, x_{n}, s\right) & =\sum_{j=1}^{q}\left|z_{j}\right|^{2}-\sum_{j=q+1}^{n-1}\left|z_{j}\right|^{2}+\operatorname{Im}\left(\sum_{j=1}^{n-1} \sum_{k=1}^{d} a_{j k} z_{j} s_{k}+\sum_{j=1}^{n-1} a_{j 0} z_{j} x_{n}\right) \\
& +\sum_{k, l=1}^{d} b_{k l} s_{k} s_{l}+\sum_{k=1}^{d} b_{k 0} s_{k} x_{n}+b_{00} x_{n}^{2}+O\left(\left|z^{\prime}\right|^{3}+\left|x_{n}\right|^{3}+|s|^{3}\right),
\end{aligned}
$$

where $b_{j k}, b_{k 0}$ and $b_{00}$ are real numbers. 
Let us now define

$\tilde{z}_{n}=z_{n}-\sum_{j=1}^{n-1} \sum_{k=1}^{d} a_{j k} z_{j} w_{k}-\sum_{j=1}^{n-1} a_{j 0} z_{j} z_{n}-\iota\left(\sum_{k, l=1}^{d} b_{k l} w_{k} w_{l}+\sum_{k=1}^{d} b_{k 0} w_{k} z_{n}+b_{00} z_{n}^{2}\right)$,

and $\tilde{x}_{n}=\operatorname{Re} \tilde{z}_{n}, \tilde{y}_{n}=\operatorname{Im} \tilde{z}_{n}$. Then we have

$$
\phi_{0}+\tilde{y}_{n}-y_{n}=\sum_{j=1}^{q}\left|z_{j}\right|^{2}-\sum_{j=q+1}^{n-1}\left|z_{j}\right|^{2}+O\left(\left|y_{n}\right|\left(\left|z^{\prime}\right|+\left|y_{n}\right|\right)+\left|z^{\prime}\right|^{3}+\left|x_{n}\right|^{3}+|s|^{3}\right) .
$$

We write the left hand side in (4.9) by using the coordinates $x_{j}, y_{j}, \tilde{x}_{n}, \tilde{y}_{n}, s_{k}, j=$ $1, \ldots, n-1, k=1 \ldots, d$, and we replace in it $\tilde{y}_{n}$ by its expression as a function of the remaining variables given by the implicit function theorem applied to $\rho^{\prime}=0$ (observe that $\left.d \rho^{\prime}\right|_{0}=\left.d \tilde{y}_{n}\right|_{0}$ ). Then we obtain a function $\Phi_{0}\left(z^{\prime}, \tilde{x}_{n}, s\right.$ ) satisfying

$$
\Phi_{0}\left(z^{\prime}, \tilde{x}_{n}, s\right)=\sum_{j=1}^{q}\left|z_{j}\right|^{2}-\sum_{j=q+1}^{n-1}\left|z_{j}\right|^{2}+O\left(\left|z^{\prime}\right|^{3}+\left|\tilde{x}_{n}\right|^{3}+|s|^{3}\right),
$$

since $\tilde{y}_{n}=y_{n}+O\left(|z|^{2}+|s|^{2}\right)$ and, on $S, \tilde{y}_{n}=O\left(\left|z^{\prime}\right|^{2}+\tilde{x}_{n}^{2}+|s|^{2}\right)$. Finally

$$
\tilde{\rho}^{\prime}:=\tilde{y}_{n}-\Phi_{0}\left(z^{\prime}, \tilde{x}_{n}, s\right)
$$

is the defining function we are looking for (after deleting the tildes).

We can now prove Theorem 1.1. We use the coordinates in Proposition 4.1, defined in an open neighborhood $\mathcal{O}$ of 0 . We can take the function $\rho^{\prime}$ in Proposition 4.1 as defining function for $S$ in $\mathcal{O}$. It will be denoted later on simply by $\rho$. Hence,

$$
\rho=y_{n}-\phi_{0}\left(z^{\prime}, x_{n}, s\right),
$$

where $\phi_{0}$ satisfies (4.4).

We therefore suppose that (1.2) holds, for some $\Omega^{\prime} \subset \Omega \subset \mathcal{O}$, and we are going to show that (3.1) cannot be true if $\mathcal{O}$ is small enough.

We begin by defining in $\mathcal{O}$ the functions

$$
h_{1}=-i z_{n}-4 \sum_{j=1}^{q}\left|z_{j}\right|^{2}-z_{n}^{2}-\sum_{k=1}^{d} w_{k}^{2}
$$

and

$$
h_{2}=i z_{n}-4 \sum_{j=q+1}^{n-1}\left|z_{j}\right|^{2}-z_{n}^{2}-\sum_{k=1}^{d} w_{k}^{2}+2 \rho \text {. }
$$


Then we take a function $\chi \in C_{c}^{\infty}\left(\Omega_{-}^{\prime}\right), \chi=1$ in some (relatively open) neighborhood of 0 in $\Omega_{-}^{\prime}$. For every $\tau>0$ we consider the forms

$$
f_{\tau}=e^{\tau h_{1}} d \bar{z}_{1} \wedge \ldots \wedge d \bar{z}_{q}
$$

and

$$
v_{\tau}=e^{\tau h_{2}} \chi d z_{1} \wedge \ldots \wedge d z_{n} \wedge d w_{1} \wedge \ldots \wedge d w_{d} \wedge d \bar{z}_{q+1} \wedge \ldots \wedge d \bar{z}_{n-1} \wedge d \rho
$$

We have

$$
f_{\tau} \in C^{\infty}\left(\Omega_{-}, \Lambda^{0, q}\right), \quad v_{\tau} \in \mathcal{J}_{c}^{\infty}\left(\Omega_{-}^{\prime}, \Lambda^{m, n-q}\right),
$$

(recall that $m=n+d$ ). We observe that

$$
\operatorname{Re} h_{1}=y_{n}-4 \sum_{j=1}^{q}\left|z_{j}\right|^{2}-x_{n}^{2}+y_{n}^{2}-|s|^{2}+O\left(\left|z^{\prime}\right|^{4}+\left|x_{n}\right|^{4}+\left|y_{n}\right|^{4}+|s|^{4}\right) \text {. }
$$

Hence, in $\mathcal{O}_{-}=\mathcal{O} \cap\left\{y_{n} \leq \phi_{0}\right\}$, for some constants $C_{1}>0, C_{1}^{\prime}>0$ we have, if $0 \leq y_{n} \ll 1$,

$$
\begin{aligned}
\operatorname{Re} h_{1} & \leq 2 y_{n}-4 \sum_{j=1}^{q}\left|z_{j}\right|^{2}-x_{n}^{2}-|s|^{2}+C_{1}\left(\left|z^{\prime}\right|^{4}+\left|x_{n}\right|^{4}+|s|^{4}\right) \\
& \leq-2\left|z^{\prime}\right|^{2}-x_{n}^{2}-|s|^{2}+C_{1}^{\prime}\left(\left|z^{\prime}\right|^{3}+\left|x_{n}\right|^{3}+|s|^{3}\right),
\end{aligned}
$$

whereas if $y_{n} \leq 0,\left|y_{n}\right| \ll 1$, for some constants $C_{2}>0, C_{2}^{\prime}>0$ it turns out that

$$
\begin{aligned}
\operatorname{Re} h_{1} & \leq y_{n} / 2-4 \sum_{j=1}^{q}\left|z_{j}\right|^{2}-x_{n}^{2}-|s|^{2}+C_{2}\left(\left|z^{\prime}\right|^{4}+\left|x_{n}\right|^{4}+|s|^{4}\right) \\
& \leq-7 \sum_{j=1}^{q}\left|z_{j}\right|^{2} / 2-\sum_{j=q+1}^{n-1}\left|z_{j}\right|^{2} / 2-x_{n}^{2}-|s|^{2}+C_{2}^{\prime}\left(\left|z^{\prime}\right|^{3}+\left|x_{n}\right|^{3}+|s|^{3}\right) .
\end{aligned}
$$

Summing up, if $\mathcal{O}$ is small enough we have

$$
\operatorname{Re} h_{1} \leq-\frac{1}{4}\left(\left|z^{\prime}\right|^{2}+x_{n}^{2}+|s|^{2}\right) \quad \text { in } \mathcal{O}_{-} .
$$


As regards $\operatorname{Re} h_{2}$, always in $\mathcal{O}_{-}$(i.e. $\rho \leq 0$ ), if $|\rho| \ll 1$, for some constant $C_{3}>0$ we have

$$
\begin{aligned}
\operatorname{Re} h_{2} & =-y_{n}-4 \sum_{j=q+1}^{n-1}\left|z_{j}\right|^{2}-x_{n}^{2}+y_{n}^{2}-|s|^{2}+2 \rho+O\left(\left|z^{\prime}\right|^{4}+x_{n}^{4}+|s|^{4}\right) \\
& =-\phi_{0}\left(z^{\prime}, x_{n}, s\right)-4 \sum_{j=q+1}^{n-1}\left|z_{j}\right|^{2}-x_{n}^{2}+y_{n}^{2}-|s|^{2}+\rho+O\left(\left|z^{\prime}\right|^{4}+x_{n}^{4}+|s|^{4}\right) \\
& =-\sum_{j=1}^{q}\left|z_{j}\right|^{2}-3 \sum_{j=q+1}^{n-1}\left|z_{j}\right|^{2}-x_{n}^{2}-|s|^{2}+\rho^{2}+\rho+O\left(\left|z^{\prime}\right|^{3}+\left|x_{n}\right|^{3}+|s|^{3}+|\rho|^{3}\right) \\
& \leq-\left|z^{\prime}\right|^{2}-x_{n}^{2}-|s|^{2}+\rho / 2+C_{2}\left(\left|z^{\prime}\right|^{3}+\left|x_{n}\right|^{3}+|s|^{3}\right) .
\end{aligned}
$$

Hence, if $\mathcal{O}$ is small enough we have $\operatorname{Re} h_{2} \leq-\left(\left|z^{\prime}\right|^{2}+x_{n}^{2}+|s|^{2}-\rho\right) / 2$ in $\mathcal{O}_{-}$. In particular there exists a constant $c>0$ such that

$$
\operatorname{Re} h_{2} \leq-c \quad \text { on the support of } d \chi .
$$

We now observe that

$$
\bar{\partial}_{M} f_{\tau}=0
$$

and

$$
\begin{aligned}
& \bar{\partial}_{M} v_{\tau}=d v_{\tau} \\
& =e^{\tau h_{2}} d \chi \wedge d z_{1} \wedge \ldots \wedge d z_{n} \wedge d w_{1} \wedge \ldots \wedge d w_{d} \wedge d \bar{z}_{q+1} \wedge \ldots \wedge d \bar{z}_{n-1} \wedge d \rho .
\end{aligned}
$$

As a consequence of (4.15), (4.16) and (4.18), for every compact $K \subset \Omega_{-}$and any $l \in \mathbb{Z}_{+}$there exist constants $C^{\prime}>0, C^{\prime \prime}>0$ such that

$$
\left\|f_{\tau}\right\|_{K, l} \leq C^{\prime} \tau^{l}, \quad\left\|\bar{\partial}_{M} v_{\tau}\right\|_{K, l} \leq C^{\prime \prime} \tau^{l} e^{-c \tau}
$$

This gives an estimate of the right hand side of (3.1) applied to the couple $f_{\tau}, v_{\tau}$. We now look at the left hand side. We have, with obvious notation,

$$
\begin{aligned}
& \int_{\rho \leq 0} f_{\tau} \wedge v_{\tau}=(-1)^{m(n-1-q)} \int_{\rho \leq 0} e^{\tau\left(h_{1}+h_{2}\right)} \chi d \bar{z}^{\prime} \wedge d z \wedge d w \wedge d \rho \\
& =(-1)^{m(n-1-q)} \int_{\rho \leq 0} e^{\tau\left(h_{1}+h_{2}\right)} \chi \operatorname{det}\left(\operatorname{Id}_{1+d}+\iota \frac{\partial \phi}{\partial\left(x_{n}, s\right)}\right) d \bar{z}^{\prime} \wedge d z^{\prime} \wedge d x_{n} \wedge d s \wedge d \rho,
\end{aligned}
$$

with $\phi:=\left(\phi_{0}, \phi_{1}, \ldots, \phi_{d}\right)$.

Moreover,

$$
\begin{aligned}
h_{1}+h_{2}= & -4\left|z^{\prime}\right|^{2} \\
& -2\left(x_{n}^{2}+|s|^{2}\right)+2 \rho+O\left(|\rho|\left(\left|x_{n}\right|+|\rho|\right)+\left|z^{\prime}\right|^{3}+\left|x_{n}\right|^{3}+|s|^{3}\right),
\end{aligned}
$$


so that

$$
\operatorname{Re}\left(h_{1}+h_{2}\right) \leq-2\left|z^{\prime}\right|^{2}-x_{n}^{2}-|s|^{2}+\rho \quad \text { in } \mathcal{O}_{-},
$$

if $\mathcal{O}$ is small enough.

We now perform the change of coordinates $\left(z^{\prime}, x_{n}, s, \rho\right) \mapsto\left(\tau^{-1 / 2} z^{\prime}, \tau^{-1 / 2} x_{n}\right.$, $\left.\tau^{-1 / 2} s, \tau^{-1} \rho\right)$ in (4.20). The new integrand function, multiplied by $\tau^{(m+n+1) / 2}$, tends to $e^{-4\left|z^{\prime}\right|^{2}-2 x_{n}^{2}-2|s|^{2}+2 \rho}$ pointwise as $\tau \rightarrow+\infty$, by (4.21) and the fact that $\chi(0)=1,\left.d \phi_{k}\right|_{0}=0, k=0, \ldots, d$. Hence, as a consequence of (4.22) and the Lebesgue dominated convergence theorem we obtain

$$
\begin{aligned}
& (-1)^{m(n-1-q)} \tau^{(m+n+1) / 2} \int_{\Omega_{-}} f_{\tau} \wedge v_{\tau} \\
& \longrightarrow \int_{\rho \leq 0} e^{-4\left|z^{\prime}\right|^{2}-2 x_{n}^{2}-2|s|^{2}+2 \rho} d \bar{z}^{\prime} \wedge d z^{\prime} \wedge d x_{n} \wedge d s \wedge d \rho \neq 0 .
\end{aligned}
$$

This fact together with (4.19) contradicts (3.1) and concludes the proof.

\section{Concluding remarks}

As an example, consider the following $C R$ structure of type $(n, 1), n \geq 2$, on $M=\mathbb{R}^{2 n+1}$, with coordinates $x_{j}, y_{j}, s, j=1, \ldots, n$, where a system of first integrals is given by $z_{j}=x_{j}+\iota y_{j}$, and $w=s+\iota \phi(z)$, with

$$
\phi(z)=\sum_{j=1}^{v}\left|z_{j}\right|^{2}-\sum_{j=v+1}^{n}\left|z_{j}\right|^{2}+O\left(|z|^{3}\right),
$$

for some integer $v \in\{0, \ldots, n\}$. Let us consider then the hypersurface $S:=\{\rho=$ $0\}$, where $\rho=y_{n}-\Phi\left(z^{\prime}\right), z^{\prime}=\left(z_{1}, \ldots, z_{n-1}\right)$, with

$$
\Phi\left(z^{\prime}\right)=\sum_{j=1}^{\mu}\left|z_{j}\right|^{2}-\sum_{j=\mu+1}^{n-1}\left|z_{j}\right|^{2}+O\left(\left|z^{\prime}\right|^{2}\right),
$$

for some $\mu \in\{1, \ldots, n-1\}$. Since $\omega_{0}=\left.d x_{n}\right|_{0}$ satisfies the hypothesis of Theorem 1.1 with $q=\mu$, it follows that, whatever the integer $v \in\{0, \ldots, n\}$ there is a neighborhood $\mathcal{O}$ of 0 such that (1.2) does not hold for every choice of $\Omega^{\prime} \subset \Omega \subset \mathcal{O}$. Moreover, as a consequence of the necessary condition in [1], the Poincaré lemma does not hold for the $\bar{\partial}_{S}$ in degree $\mu$ (and $n-1-\mu$, if $\mu<n-1$ ) at 0 . However, from the results in [2] it follows that the Poincare lemma holds true for the $\bar{\partial}_{M}$ at 0 in any degree $1 \leq q^{\prime} \leq n, q^{\prime} \neq v, q^{\prime} \neq n-v$.

Finally we want to present a necessary condition for (1.2) to hold in top degree, namely with $q=n$ (notice that the compatibility condition $\bar{\partial}_{M} f=0$ is now automatically satisfied). In this case one can directly make use of the known necessary condition for the existence of the Poincaré lemma in top degree for the $\bar{\partial}_{M}$ (see $[1,17])$. We emphasize that we do not suppose here that $M$ is locally embeddable. 
Proposition 5.1. Let $M$ be a $C R$ manifold of type $(n, d)$, and suppose that the Levi form of $M$ at some characteristic point $\left(0, \omega_{0}\right) \in T^{0} M$ is definite. Then for every non-characteristic hypersurface $S \subset M$ through 0, (1.2) does not hold for any choice of the open neighborhoods $\Omega^{\prime} \subset \Omega \subset M$ of 0 . The same holds with $\Omega_{-}, \Omega_{-}^{\prime}$ replaced by $\Omega_{+}=\Omega \cap\{\rho \geq 0\}$ and $\Omega_{+}^{\prime}=\Omega^{\prime} \cap\{\rho \geq 0\}$.

Proof. The proof is by contradiction. From (1.2) with $q=n$ it follows that for every open set $V \subset \subset \operatorname{int}\left(\Omega_{-}^{\prime}\right)$ the system $\bar{\partial}_{M}$ admits a solution $u \in \mathcal{D}^{\prime}\left(V, \Lambda^{0, n-1}\right)$ in $V$ for every $f \in C^{\infty}\left(\operatorname{int}\left(\Omega_{-}^{\prime}\right), \Lambda^{0, n}\right)$. Indeed, it suffice to consider any $\tilde{f} \in$ $C^{\infty}\left(\Omega_{-}, \Lambda^{0, n}\right)$, with $\tilde{f}=f$ in $V$, and to solve $\bar{\partial}_{M} \tilde{u}=\tilde{f}$ with $\tilde{u} \in \mathcal{A}^{\prime}\left(\Omega_{-}^{\prime}, \Lambda^{0, n-1}\right)$. Then $u:=\left.\tilde{u}\right|_{V} \in \mathcal{D}^{\prime}\left(V, \Lambda^{0, n-1}\right)$ satisfies $\bar{\partial}_{M} u=f$ in $V$.

However the Levi form of $M$ will be certainly definite at some characteristic point $\left(x_{0}, \tilde{\omega}_{0}\right) \in T^{0} M, \tilde{\omega}_{0} \neq 0$, with $x_{0} \in \operatorname{int}\left(\Omega_{-}^{\prime}\right)$ sufficiently near 0 , and this contradicts Corollary VIII.2.2 of [17]. The same arguments apply, of course, to the other side of $S$.

\section{References}

[1] A. Andreotti, G. Fredricks and M. Nacinovich, On the absence of the Poincaré lemma in tangential Cauchy-Riemann complexes, Ann. Scuola Norm. Sup. Pisa Cl. Sci. 8 (1981), 365-404.

[2] A. Andreotti and C. D. Hill, E.E. Levi convexity and the Hans Lewy problem, I and II, Ann. Scuola Norm. Sup. Pisa Cl. Sci. 26 (1972), 325-363, 747-806.

[3] P. D. Cordaro and J. Hounie, Local solvability for a class of differential complexes, Acta Math. 187 (2001), 191-212.

[4] P.D. Cordaro and F. Treves, "Hyperfunctions on Hypo-Analytic Manifolds", Annals of Mathematics Studies, Vol. 136. Princeton University Press, Princeton, NJ, 1994.

[5] S.-C. CHEN and M.-C. SHAw, "Partial Differential Equations in Several Complex Variables", Studies in Advanced Mathematics, Vol. 19, Amer. Math. Soc., International Press, Providence, RI, 2001.

[6] G. B. FOLLAND and E.M. STEIN, Estimates for the $\bar{\partial}_{b}$ complex and analysis on the Heisenberg group, Comm. Pure Appl. Math. 27 (1974), 429-522.

[7] C. D. HiLl and M. NACINOVICH, On the failure of the Poincaré lemma for $\bar{\partial}_{M} I I$, preprint.

[8] L. Hörmander, Differential operators of principal type, Math. Ann. 140 (1960), 124 146.

[9] M. KASHIWARA and P. SCHAPIRA, A vanishing theorem for a class of systems with simple characteristics, Invent. Math. 82 (1985), 579-592.

[10] H. LEWY, An example of a smooth linear partial differential equation without solution, Ann. of Math. 66 (1957), 155-158.

[11] R. B. Melrose, "Differential Analysis on Manifolds with Corners", book in preparation, available at www.math.mit.edu/ $\sim \mathrm{rbm} /$ book.html.

[12] V. Michel, Sur la régularité $C^{\infty} d u \bar{\partial}$ au bord d'un domaine de $\mathbb{C}^{n}$ dont la forme de Levi a exactement s valeus propres négatives, Math. Ann. 295 (1993), 135-161.

[13] M. Nacinovich, Poincaré lemma for tangential Cauchy-Riemann complexes, Math. Ann. 268 (1984), 449-471.

[14] M. NACINOVICH, On strict Levi q-convexity and q-concavity on domains with piecewise smooth boundaries, Math. Ann. 281 (1988), 459-482. 
[15] M. M. Peloso and F. RICCI, Tangential Cauchy-Riemann equations on quadratic CR manifolds, Atti Accad. Naz. Lincei Cl. Sci. Fis. Mat. Natur. Rend. Lincei 13 (2002), 125134.

[16] F. Treves, "Homotopy Formulas in the Tangential Cauchy-Riemann Complex", Memoirs, Vol. 87, No. 434, Amer. Math. Soc., Providence, RI, 1990.

[17] F. Treves, "Hypo-analytic Strucures: Local Theory", Princeton University Press, Princeton, 1992.

[18] F. TREVES, A treasure trove of geometry and analysis: The hyperquadric, Notices Amer. Math. Soc. 47 (2000), 1246-1256.

Dipartimento di Matematica

Politecnico di Torino

Corso Duca degli Abruzzi, 24

10129 Torino, Italy

fabio.nicola@polito.it 